\title{
Epidemiología, vigilancia y control de enfermedades tropicales
}

BACTERIAS

\section{Impacto de una actividad formativa en los conocimientos, actitudes y percepciones sobre tuberculosis en los estudiantes de medicina de una universidad colombiana}

Diana M. Castañeda-Hernández, Álvaro MondragónCardona, Carlos Felipe Campo-Betancourth, Daniel Tobón-García, Verónica Alzate-Carvajal, Carlos Eduardo Jiménez-Canizales, Alfonso J. Rodríguez Programa de Tuberculosis y Lepra, Secretaría de Salud y Seguridad Social de Pereira; Universidad Tecnológica de Pereira, Pereira, Colombia

Introducción. La tuberculosis continúa siendo una de las principales enfermedades tropicales en términos de morbimortalidad. De allí, la importancia de mejorar su prevención mediante diferentes estrategias, incluida la educación médica continua.

Materiales y métodos. En el marco del día mundial de la lucha contra la tuberculosis, se realizó un simposio educativo que incluyó charlas y una obra de teatro sobre diferentes aspectos de la enfermedad con una duración de 280 minutos. Antes de iniciar se aplicó una encuesta para evaluar los conocimientos actitudes y percepciones sobre la tuberculosis, basada en la guía para encuestas ACMS, de Stop TB Partnership de la Organización Mundial de la Salud. Al finalizar, se aplicó de nuevo. Se compararon las diferencias entre los resultados antes y después con la prueba $\mathrm{X}^{2}$ y confianza del 95\%.

Resultados. Se evaluaron 109 universitarios antes de iniciar y 102 después ( $n=211$ encuestas). La primera pregunta fue: "¿Cuán serio percibe usted el problema de la tuberculosis en su comunidad?". En la encuesta previa, sólo 50,9 \% lo consideró un problema muy serio. En la posterior se incrementó a $94,1 \%\left(x^{2}=49,602 ; p<0,001\right)$. En la encuesta previa, 31,5\% lo consideró como algo serio, 2,8\% no tan serio, 8,3 \% no sabía y 6,5\% no contestó. En la posterior, el restante 5,9 \% correspondió sólo a considerarlo como algo serio.

En general, se observó una mejoría significativa sobre los conocimientos actitudes y percepciones en síntomas, transmisión, prevención y curación, que sentiría al ser diagnosticado y otros aspectos evaluados $(p<0,05)$.
Conclusiones. Este tipo de abordajes, especialmente a nivel comunitario, debe ser replicado, con el fin de mejorar los conocimientos actitudes y percepciones de la población sobre la tuberculosis, no sólo la población universitaria, sino también la general. Se requiere impartir mayor información a las poblaciones afectadas y a sus familias sobre las formas de transmisión, para mitigar los mitos y el estigma que es, también, prevalente.

\section{Prevalencia de leptospirosis humana y porcina en el área rural de Mercaderes, Cauca}

Luis Reinel Vásquez¹, Piedad M. Agudelo-Flórez², Julio C. Giraldo ${ }^{3}$, Diego Vergara ${ }^{4}$, Diana Samper ${ }^{1}$, Diana

L. Nieto ${ }^{1}$, Omar Ramos ${ }^{1}$, Lina M. Bonilla ${ }^{1}$, Víctor H. Campo 5

1 Centro de Estudios en Microbiología y Parasitología, Departamento de Medicina Interna, Facultad de Ciencias de la Salud, Universidad del Cauca, Popayán, Colombia

2 Instituto Colombiano de Medicina Tropical, Universidad CES, Sabaneta, Colombia

3 Grupo de Investigación en Parasitología y Microbiología Tropical, Universidad INCCA, Bogotá, D.C., Colombia

4 Sisimpro, Facultad de Ciencias Agropecuarias, Universidad del Cauca, Popayán, Colombia

5 FTP, Departamento de Ciencias Fisiológicas, Facultad de Ciencias de la Salud, Universidad del Cauca, Popayán, Colombia

Introducción. La leptospirosis es una zoonosis emergente de importancia mundial. En el departamento del Cauca se desconocen la situación de la enfermedad y los factores de riesgo que condicionan la exposición a la infección en la población humana.

Métodos. Para determinar la seroprevalencia de leptospirosis en humanos y en porcinos se realizó un estudiodescriptivo. Seobtuvounamuestradesangre a habitantes y cerdos. Se determinaron anticuerpos anti-Leptospira por inmunofluorescencia indirecta y microaglutinación. Se realizó una intervención comunitaria e institucional y se llevó a cabo una evaluación posterior para determinar los cambios en la prevalencia porcina. Se hizo una encuesta estructurada de factores de riesgo. Se utilizó el 
paquete SPSS® para el análisis estadístico.

Resultados. La seroprevalencia de leptospirosis fue de $6 \%$ (40/665), mientras que en los porcinos fue de $22,9 \%(38 / 166)$ en el primer muestreo y de $14,8 \%(19 / 166)$ en el segundo muestreo. Las serovariedades más prevalentes en las dos poblaciones fueron Bratislava e Icterohemorragiae. Los resultados seropositivos para leptospirosis en humanos se relacionó con la calidad del agua $(p=0,048)$; algunos factores fueron protectores, como la procedencia y la ocupación. Se observó una disminución de la leptospirosis porcina con una segunda evaluación serológica, con una tendencia similar en el muestreo del área periurbana.

Conclusiones. Los resultados señalan que en la zona rural del municipio se presentan las condiciones ambientales y ocupacionales apropiadas para que la enfermedad se perpetúe. Todo esto está condicionado por la falta de saneamiento ambiental que ocasiona un aumento de roedores en las zonas aledañas a las viviendas y a las fuentes de agua para consumo humano. Con las alianzas hechas a nivel institucional, la utilización de IAP y la participación comunitaria se observó una disminución en los resultados positivos de leptospirosis porcina. Se debe continuar con las actividades de intervención e investigación para dar sostenibilidad a las actividades recomendadas para el control de esta zoonosis.

Este trabajo se realizó en el marco de un proyecto de Colciencias (contrato 307), cofinanciado por la Universidad del Cauca, la Universidad INCCA y la Universidad CES; además, con recursos proporcionados por la Vicerrectoría de Investigaciones de la Universidad del Cauca con código id2287.

\section{Perfil epidemiológico y clínico de los pacientes con leptospirosis, Urabá, Antioquia}

Margarita Arboleda, Piedad Agudelo-Flórez, Gabriel Jaime Parra, Carlos Tangarife, Jesús Ochoa Instituto Colombiano de Medicina Tropical, Universidad CES, Apartadó y Sabaneta, Colombia Grupo de Epidemiología, Universidad de Antioquia, Medellín, Colombia

Introducción. La leptospirosis representa un problema de salud pública y es una causa importante de mortalidad en la región de Urabá; usualmente, los pacientes con síndrome febril agudo y manifestaciones hemorrágicas se diagnostican como dengue, el principal diagnóstico diferencial de leptospirosis, pero el tratamiento de ambas enfermedades es diferente.

El presente estudio pretendió caracterizar el perfil clínico y epidemiológico de los pacientes con cuadros leves y graves de leptospirosis, procedentes de los municipios del eje bananero (Chigorodó, Carepa, Apartadó y Turbo).

Materiales y métodos. Se llevó a cabo un estudio de casos y controles, de 153 pacientes con leptospirosis, 66 casos con presentaciones graves y 87 controles con formas leves o moderadas. Los casos se captaron por hospitalización, y los controles, en consulta externa o en los servicios de urgencias. El diagnóstico de leptospirosis se hizo por la detección de anticuerpos IgM e IgG en muestras pareadas, o por el aislamiento de leptospira en sangre. Se construyeron los perfiles de ambos grupos mediante análisis de correspondencias múltiples.

Resultados. El $51 \%$ de los casos (33/66) y el $28 \%(24 / 87)$ de los controles presentaron manifestaciones hemorrágicas. El perfil preliminar de los casos correspondía a pacientes con manifestaciones hemorrágicas, sin antecedentes de episodios previos de leptospirosis; el perfil preliminar de los controles, a pacientes sin manifestaciones hemorrágicas que no requirieron remisión al tercer nivel de atención. No hubo diferencias en las manifestaciones generales (fiebre, cefalea, osteomialgias y malestar) y síntomas clásicos (dolor en las pantorrillas, dolor lumbar e ictericia) entre los casos y los controles.

Conclusiones. En las áreas endémicas para varias enfermedades infecciosas se debe tener en cuenta a la leptospirosis como diagnóstico diferencial en los pacientes con cuadro febril agudo y manifestaciones hemorrágicas, para orientar una conducta terapéutica oportuna (antibioticoterapia) y evitar las complicaciones y la muerte.

Estado de la coinfección VIHtuberculosis en el municipio de Armenia: 10 años de experiencia

Nelson E. Arenas ${ }^{1,2}$, Natalia Ramírez-Girón¹, Gilberto González-López 1 , Silvana Rubertone ${ }^{2}$, Sandra M. Coronado ${ }^{1}$, Adriana M. García ${ }^{3}$, Jorge Enrique GómezMarín $^{4}$, Liliana Quintero²

1 Grupo de Investigación en Ciencias Biomédicas, Centro de Investigaciones Biomédicas, Universidad del Quindío, Armenia, Colombia

2 Programa de Vigilancia Epidemiológica, Secretaría de Salud, Armenia, Colombia

3 Programa de Tuberculosis-VIH, Hospital Universitario San Juan de Dios, Armenia, Colombia 
4 Grupo de Parasitología Molecular, Centro de Investigaciones Biomédicas, Universidad del Quindío, Armenia, Colombia

Introducción. La tuberculosis representa una de las principales coinfecciones en pacientes diagnosticados con el virus de la inmunodeficiencia humana (VIH). Así, cuando el procedimiento diagnóstico no es oportuno, el desenlace puede ser fatal por la inminente progresión de la enfermedad.

El objetivo de este estudio fue evaluar los aspectos clínicos, epidemiológicos y sociodemográficos de los pacientes enfermos con tuberculosis-VIH en el periodo comprendido entre 2000 y 2010.

Materiales y métodos. Se realizó un estudio descriptivo retrospectivo entre los años 2000 y 2010 en Armenia. Se tomaron los datos sociodemográficos de las fichas de notificación al Sivigila de tuberculosis-VIH, las actas de visitas domiciliarias, las tarjetas individuales de tratamiento y los certificados de defunción. Los criterios de inclusión fueron la procedencia de Armenia y el diagnóstico confirmado de tuberculosis-VIH. Los datos se analizaron en el programa Epilnfo, versión 3.5.3.

Resultados. Se incluyeron 113 pacientes con diagnóstico confirmado de coinfección tuberculosisVIH (prevalencia global, 6,7\%).
La edad promedio fue de 34,3 años (rango, 11 a 68 años). La edad media en tuberculosis extrapulmonar fue de 31,5 (rango, 20 a 68 años), y 33,5 (rango, 27 a 59 años) en tuberculosis pulmonar $(p=0,5)$. El sexo que predominó de manera estadísticamente significativa fue el masculino con $91 \%(51 / 56)$ en las formas extrapulmonares y de $75 \%$ en las pulmonares (42/56); el odds ratio (OR) de la tuberculosis extrapulmonar en mujeres fue de 0,2 $\left(\mathrm{IC}_{95 \%} 0,0-0,8, \mathrm{p}=0,02\right)$.

La tuberculosis pulmonar con 49,5 \% $(n=56)$, predominó sobre la tuberculosis extrapulmonar con 46,4 \% $(n=46)$ de los casos; los cuadros clínicos mixtos, 6,1 \% $(n=7)$ y la infección por otras micobacterias, $3,5 \% \quad(n=4)$. Las formas extrapulmonares más prevalentes fueron: tuberculosis ganglionar (50\%, 23 casos) seguida de tuberculosis meníngea (11 casos, 23,9\%).

Conclusiones. Los resultados de este estudio evidencian la importancia de implementar los programas de VIH y tuberculosis en los diferentes niveles de atención y la programación de actividades que se deben realizar en cada instancia para garantizar la búsqueda de $\mathrm{VIH}$ en pacientes con tuberculosis y viceversa. 


\section{Epidemiología, vigilancia y control de enfermedades tropicales}

Cisticercosis

\section{Cisticercosis humana en pacientes con diagnóstico de epilepsia en un centro de salud de Popayán, Cauca}

Jagui Vergara ${ }^{1}$, Martín Ortega', Luis Reinel Vásquez¹, Juan Carlos Casas², Julio C. Giraldo ${ }^{3}$

1 Centro de Estudios en Microbiología y Parasitología, Departamento de Medicina Interna, Facultad de Ciencias de la Salud, Universidad del Cauca, Popayán Colombia

2 Liga Colombiana contra la Epilepsia, Capítulo Cauca, Popayán, Colombia

3 Grupo de investigación en Parasitología y Microbiología Tropical, Universidad INCCA de Colombia, Bogotá, D.C., Colombia

Introducción. El complejo teniasis/cisticercosis es causado por Taenia solium, es considerada como un problema de salud pública y es una de las parasitosis que se asocia como causa de epilepsia. El departamento del Cauca no está exento de presentar esta parasitosis.

El objetivo del proyecto fue determinar la prevalencia de cisticercosis humana en pacientes con diagnóstico de epilepsia en un centro hospitalario de tercer nivel durante el 2007.

Métodos. Se realizó un estudio retrospectivo, durante el primer semestre de 2007 basado en la revisión de historias clínicas en un centro hospitalario de tercer nivel. Se aplicararon encuestas basadas en las historias clínicas que incluían datos sociodemográficos y clínicos.

Resultados. Se revisaron 111 historias clínicas que se encontraban en el área deconsulta externa del Servicio de Neurología de la institución hospitalaria en 2007. De ellas, 53,2 \% eran de pacientes de sexo masculino, y la edad promedio fue de 33,2 años. El 55 \% procedían del área rural, y Popayán fue el municipio de mayor frecuencia. En 17,1 \% se encontraron anticuerpos anti-cisticerco. El 51,4 $\%$ presentaba crisis generalizadas y $31,5 \%$ crisis focales.

Conclusiones. Este es primer estudio realizado en el departamento del Cauca, en el que se evalúa la cisticercosis en pacientes con diagnóstico de epilepsia. El hallazgo de 111 historias clínicas con diagnóstico de epilepsia en un solo centro hospitalario debería llamar la atención a las autoridades de salud y a aquellas encargadas de realizar actividades de promoción de una vida saludable y prevención de este tipo de enfermedades, ya que es un síndrome que todavía en Colombia tiene causas "divinas" o "de demonios".

Como lo sugiere la OMS/OPS, debe actualizarse la epidemiología de la epilepsia junto con la de la cisticercosis y así implementar estrategias de vigilancia en el contexto local y nacional.

$$
\text { - } \bullet
$$

\section{Conocimientos, actitudes y prácticas de los porcicultores y comercializadores sobre la teniasis/ cisticercosis en el municipio El Guamo, departamento del Tolima, Colombia}

Julio César Giraldo", Reinel Vásquez², Diana Nieto², Angie Manrique $^{3}$, Madelyn Romero ${ }^{1}$

${ }^{1}$ Grupo de Investigación en Parasitología y Microbiología Tropical, Universidad Incca de Colombia, Bogotá, D.C., Colombia

2 Centro de Estúdios en Microbiología y Parasitología, Departamento de Medicina Interna, Facultad de Ciencias de la Salud, Universidad del Cauca, Popayán, Colombia

Introducción. La cisticercosis es una enfermedad parasitaria endémica en nuestro país, de la cual, hasta el momento, no se han tomado medidas de salud que nos permitan el control de la misma.

Se realizó un estudio cualitativo que permitió evidenciar las prácticas y crianzas de cerdos de los pequeños porcicultores en el área urbana del municipio El Guamo, departamento del Tolima.

Materiales y métodos. Se emplearon encuestas estructuradas en las que se evaluaron las condiciones de vivienda, las características sociodemográficas, los hábitos de higiene y los síntomas presentes asociados al agente parasitario; además, se realizó un estudio cuantitativo al azar de 48 porcinos que fueron sacrificados en el matadero municipal de junio de 2010 a marzo de 2011 mediante la prueba de tamización ELISA con una fracción antigénica de 53 kDa para determinar la frecuencia de anticuerpos contra el metacestodo con un nivel de confianza del 95\%. Las variables sociodemográficas y sintomatológicas se analizaron 
mediante el cálculo de la frecuencia simple y el empleo de tablas de contingencia de dos por dos con el programa estadístico SPSS $\AA$, v. 15.

Resultados. La seroprevalencia de anticuerpos anticisticerco en los cerdos sacrificados para este período fue de $8,33 \%$ y los factores de riesgo que se encontraron asociados fueron las condiciones de higiene personal en algunas poblaciones, como la defecación al aire libre $(3,20, \%)$, el deficiente lavado de manos (28,82\%), el consumo de carnes o de verduras crudas o sin lavar $(2,70 \%)$, la cría de cerdos en condiciones poco tecnificadas $(18,50$ $\%)$, con alimentación inadecuada $(19,60 \%)$, con malas condiciones sanitarias y de bioseguridad $(15,20 \%)$, el transporte y la comercialización de la carne $(58,1 \%)$; todo esto hace que esta cadena de transmisión se perpetúe. De igual manera, las medidas de control tomadas en el matadero no son suficientes para prevenir esta problemática.

Conclusión. La modificación de las condiciones sanitarias en esta población constituye una prioridad de salud pública.

\section{Evolución de la cisticercosis diagnosticada por el laboratorio clínico en el departamento del Cauca}

Laura Cristancho', Luis Reinel Vásquez² ${ }^{2}$ Tomás O.

Zamora $^{2}$, Juan C. Casas ${ }^{3}$, Julio C. Giraldo ${ }^{4}$

1 Acemcauca, Facultad de Ciencias de la Salud, Universidad del Cauca, Popayán, Colombia

2 Centro de Estudios en Microbiología y Parasitología, Departamento de Medicina Interna, Facultad Ciencias de la Salud, Universidad del Cauca, Popayán, Colombia

${ }^{3}$ Liga Colombiana de la Epilepsia, Capítulo Cauca, Popayán, Colombia

${ }^{4}$ Grupo de Investigación en Parasitología y Microbiología Tropical, Universidad INCCA, Bogotá, D.C., Colombia

Introducción. La cisticercosis causada por Taenia solium es un problema de salud pública en el departamento del Cauca, y todavía no se vislumbra un cambio a corto o mediano plazo. Se hizo el diagnóstico serológico de los anticuerpos anticisticercodepacientesvaloradosenellaboratorio del Grupo de Investigación en Parasitología y Microbiología Tropical de la Universidad INCCA y el Laboratorio de Microbiología y Parasitología de la Facultad de Ciencias de la Salud de la Universidad del Cauca, en 2010 y 2011.

Materiales y métodos. Se realizó un estudio descriptivo del 2010 al 2011 de los pacientes que son atendidos en el Laboratorio de Microbiología y
Parasitología de la Facultad de Ciencias de la Salud de la Universidad del Cauca. Se utilizó la técnica ELISA con la fracción de 53 kDa (sensibilidad, 100 $\%$, y especificidad, $99,1 \%$,), realizada por el Grupo de Investigación en Parasitología y Microbiología tropical de la Universidad INCCA para determinar los anticuerpos anticisticerco, acompañada de una encuesta estructurada. Se obtuvo consentimiento informado.

Resultados. Se procesaron 20 encuestas, 12 del año 2010, todos eran pacientes del departamento del Cauca. El 52,6 \% presentaba anticuerpos anticisticerco. El promedio de edad fue de 39 años y la población se encontraba entre los 14 y los 74 años. El $40 \%$ presentó crisis convulsiva y $80 \%$ manifestaba cefalea.

Discusión. En el departamento del Cauca se siguen presentando casos de cisticercosis humana con importantes complicaciones clínicas; se hace un llamado a las autoridades de salud en torno a esta parasitosis.

\section{Prevalencia de cisticercosis humana en el area rural de Mercaderes, Cauca}

Luis Reinel Vásquez ${ }^{1}$, Piedad M. Agudelo-Flórez², Julio C. Giraldo ${ }^{3}$, Diego Vergara ${ }^{4}$, Diana Samper ${ }^{1}$, Diana L. Nieto', Omar Ramos ${ }^{1}$, Lina M. Bonilla ${ }^{1}$, Víctor H. Campo $^{5}$

1 Centro de Estudios en Microbiología y Parasitología, Departamento de Medicina Interna, Facultad Ciencias de la Salud, Universidad del Cauca, Popayán, Colombia

2 Universidad CES, Medellín, Colombia

${ }^{3}$ Grupo de Investigación en Parasitología y Microbiología Tropical, Universidad INCCA de Colombia, Bogotá, D.C., Colombia

${ }^{4}$ Sisimpro, Facultad de Ciencias Agropecuarias, Universidad del Cauca, Popayán, Colombia

${ }^{5}$ FTP, Departamento de Ciencias Fisiológicas, Facultad de Ciencias de la Salud, Universidad del Cauca, Popayán, Colombia

Introducción. El complejo teniasis/cisticercosis es un problema de salud pública en países de América Latina como Colombia, debido a diversos factores como la presencia de pacientes con tenias, la crianza no tecnificada del cerdo y la deficiencia sanitaria y educativa, entre otros.

Se realizó un estudio seroepidemiológico para determinar la prevalencia de cisticercosis humana en el área rural de Mercaderes, Cauca.

Métodos Se hizo un muestreo en el área rural en población humana con consentimiento informado y se determinaron los anticuerpos anticisticerco por medio de la técnica ELISA con la fracción de 53 
kDa. Se aplicó una encuesta estructurada.

Resultados. Se realizaron 665 serologías en la determinación de anticuerpos anticisticerco resultando positivas 28,4\% (189), los resultados seropositivos se relacionaron con la procedencia, la fuente de agua y el lugar donde se bañaban, entre otras. Se adelantaron discusiones por medio de grupos focales en las que se aclaró el ciclo de vida del parásito.

Conclusiones. El complejo teniasis/cisticercosis es un problema de salud pública en Mercaderes; se deben mejorar las actividades de control de esta parasitosis por parte de las autoridades locales con una mayor presencia e inversión.

Este trabajo se realizó en el marco de un proyecto de Colciencias (contrato 307), cofinanciado por la Universidad del Cauca, la Universidad INCCA y la Universidad CES; además, con recursos proporcionados por la Vicerrectoría de Investigaciones de la Universidad del Cauca con código id2287.

\section{Neurocisticercosis, un problema que sigue afectando a nuestra población caucana.}

Víctor Hugo Vivas", Luis Reinel Vásquez¹, Tomás O.

Zamora ${ }^{1}$, Julio C. Giraldo²

1 Centro de Estudios en Microbiología y Parasitología, Departamento de Medicina Interna, Facultad de Ciencias de la Salud, Universidad del Cauca, Popayán Colombia

2 Grupo de Investigación en Parasitología y Microbiología Tropical, Universidad INCCA, Bogotá, D.C., Colombia

Introducción. Nuestra población caucana sigue siendo afectada de una manera importante por Taenia solium, ya que los cisticercos afectan el sistema nervioso central.

Se presenta un caso clínico de neurocisticercosis del primer semestre del 2011 y se hace una revisión de esta parasitosis para el departamento del Cauca.
Caso clínico. Se presenta el caso de un paciente de 29 años, de sexo masculino, procedente de zona rural del departamento del Cauca, que consultó al nivel I por cefalea global intensa y vómito de tres meses de evolución, acompañado de trastornos de la marcha y de la conducta que se agudizaron en la última semana.

Fue remitido al nivel III, y en la tomografía se informó hidrocefalia supratentorial y edema subependimario; se decidió colocarle una válvula de derivación ventrículo-peritoneal.

El trascurso de la evolución fue desfavorable con escala de Glasgow en descenso, paraplejía y amaurosis bilateral; se reportó colapso ventricular, y se solicitaron exámenes complementarios, cuyos resultados fueron: negativo para $\mathrm{VIH}$; punción lumbar, negativa; VDRL, negativo; en la resonancia se observó aracnoiditis que comprometía todos los segmentos de la columna y mielitis a la altura de T3 y T4; ELISA anticisticerco, positiva con títulos elevados $(0,944)$ que confirmó el diagnóstico de neurocisticercosis.

Se inició tratamiento con dexametasona por cinco días y albendazol por 14; el paciente no se recuperó de la paraplejía, ni de la amaurosis; la escala de Glasgow se recuperó, 15/15, y la cefalea desapareció. Fue dado de alta después de un mes de haber sido hospitalizado con el servicio de atención en casa.

Discusión. Este es uno de los casos que demuestra que esta parasitosis continúa presente en nuestra población caucana; es un problema de salud pública que se podría evitar con actividades eficaces de promoción y prevención que hagan hincapié en que la culpa de esta enfermedad no es del cerdo y enfatizando que las complicaciones y secuelas de la neurocisticercosis son fatales, cada vez más frecuentes en los pacientes y costosas para las entidades de salud. 


\title{
Epidemiología, vigilancia y control de enfermedades tropicales
}

\author{
ENFERMEDAD DE CHAGAS
}

\section{Enfermedad de Chagas: la importancia epidemiológica de estudiar grupos familiares}

Inés Zulantay, Daniel Ramos, Eduardo Sepúlveda, Patricio Thieme, Alfredo Araya, Sergio Astete, María Aguilera, Werner Apt

Laboratorio de Parasitología Básico-Clínico, Programa

Biología Celular y Molecular, ICBM, Facultad de

Medicina, Universidad de Chile; Hospital de Salamanca, Hospital de Los Vilos, Servicio de Salud Coquimbo, IV Región, Chile

Introducción. La enfermedad de Chagas constituye un problema de salud pública y su control incluye la pesquisa de nuevos casos para su adecuado manejo

Objetivo. Determinar el número de personas infectadas por Trypanosoma cruzi en grupos familiares de una zona endémica de Chile, a partir de un caso índice.

Materiales y métodos. Se consideró caso índice a las mujeres con enfermedad de Chagas confirmada serológicamente mediante ELISA e IFI-IgG durante su embarazo.

Resultados. Durante los años 2007 a la fecha, ha sido posible estudiar las familias de 92 casos índice. Cinco hijos de estas madres son casos congénitos, con estudio al nacimiento y confirmación serológica después de los 18 meses de vida. Nueve hermanos maternos están infectados, con edades que fluctúan entre los 3 y los 15 años. En relación con las abuelas maternas, en 70 de ellas la infección ha sido confirmada. Finalmente, en 85 hermanos maternos se pudo establecer la infección por T. cruzi. Este último grupo presenta dificultades de estudio por migraciones a otras áreas, generalmente, en búsqueda de mejores oportunidades laborales.

El resultado global indica que en las familias estudiadas, considerando el caso índice, al menos 248 miembros de ellas tienen enfermedad de Chagas, con un promedio de 2,83 infectados por familia. Parte de estos infectados ya han sido tratados con nifurtimox.

Conclusión. El estudio familiar en torno a un caso índice podría modificar las estimaciones de prevalencia de la infección por $T$. cruzi en nuestras zonas endémicas.
Financiamiento: proyectos Fondecyt 1100768 y 1080445

\section{Enfermedad de Chagas en áreas endémicas: la urgente necesidad de educación en los equipos de salud}

Inés Zulantay, Sebastián Galafé, Alberto Torres, Claudio Valencia, Carolina Rivera, Natalia Rossel, Werner Apt Laboratorio de Parasitología Básico-Clínico, Programa de Biología Celular y Molecular, ICBM, Facultad de Medicina, Universidad de Chile;

Departamento de Salud, municipalidad de Combarbalá, Hospital de Salamanca, Hospital de Illapel, Hospital de Los Vilos, SS Coquimbo, IV Región, Chile

Objetivo. Establecer, mediante la aplicación de una herramienta diagnóstica, el grado de conocimiento sobre la enfermedad de Chagas en los equipos de salud de un área endémica de Chile.

Materiales y métodos. Se diseñó una encuesta diagnóstica de carácter voluntario y anónimo de 41 preguntas de selección múltiple con respuestaúnica que incluyó aspectos biológicos, epidemiológicos, clínicos, diagnósticos, terapéuticos y preventivos sobre la enfermedad de Chagas. En la encuesta, validada por expertos, se solicitó indicar el tipo de centro asistencial (posta, hospital o consultorio) y profesión (médico, enfermera, matrona, tecnólogo médico, paramédico u otro). Debido a la alta rotación de personal, se estableció como criterio de inclusión la permanencia mínima de 1 año en cada centro asistencial.

Aceptaron contestar voluntariamente la encuesta 138 personas (37 médicos, 13 matronas, 10 enfermeras, 4 tecnólogos médicos, 72 paramédicos, 1 nutricionista, 1 psicólogo), 39 procedentes de la Comuna I, 19 de la Comuna II, 27 de la Comuna III, 24 de la Comuna IV, 18 de la Comuna V y 11 profesionales que conforman equipos de manejo de la enfermedad de Chagas en la zona, la mayoría nuevos en este rol.

Resultados. La evaluación global, sin considerar estratificación de competencias mínimas, indica que no sobrepasaron el $60 \%$ de respuestas correctas el $69,2 \%, 89,5 \%, 92,6 \%, 100 \%$ y $94,4 \%$ de los encuestados de las Comunas I-V, respectivamente, $y$ el $63,6 \%$ del equipo de Chagas. El menor y 
mayor porcentaje de respuestas correctas fue de $2 \%$ y $72 \%$, respectivamente.

Conclusión. Se confirma la urgente necesidad de implementar acciones continuas de educación, información y comunicación sobre la enfermedad de Chagas, dirigidas a los equipos de salud de las zonas endémicas con el fin de otorgar al individuo infectado, adecuada información y atención integral. La siguiente etapa del estudio es la intervención educativa de acuerdo con el diagnóstico estratificado establecido.

Financiamiento: proyectos Fondecyt 1100768 y 1080445

\section{Etnometodología para la investigación de la enfermedad de Chagas en comunidades indígenas wiwa de la Sierra Nevada de Santa Marta, 2010}

Leonardo Alberto Ríos-Osorio, José Crespo-González, Mario Augusto Zapata-Tamayo

Grupo de Investigación Salud y Sostenibilidad, Escuela de Microbiología, Universidad de Antioquia, Medellín, Colombia

Introducción. El objetivo de este trabajo fue establecer la prevalencia de la enfermedad de Chagas en comunidades wiwa de la Sierra Nevada de Santa Marta, trascendiendo el modelo de investigación biomédica sustentado en el paradigma positivista e involucrando la dimensión sociocultural y ambiental que caracteriza este fenómeno, desde la sostenibilidad como un nuevo paradigma de las ciencias.

Materiales y métodos. Se realizó un muestreo probabilístico de 15 comunidades wiwa asentadas en la zona de San Juan del César, La Guajira; se realizaron los procedimientos biomédicos definidos para investigaciones epidemiológicas; paralelamente, se realizaron procedimientos culturales desde el saber tradicional de las comunidades wiwa, garantizando la armonía de las comunidades ante la agresión biomédica de su espacio ambiental, social y cultural.

Resultados. Se obtuvo un prevalencia de 33,5 $\%$, concordante con las cifras de enfermedad de Chagas encontradas en las otras vertientes de la sierra, reflejando condiciones similares que predisponen a la presencia de la enfermedad. Se estableció cómo esta enfermedad es inexistente en el sistema médico tradicional de los wiwa, y sólo el insecto vector es reconocido aunque no considerado como agente perturbador de la salud de las comunidades. A partir de la consideración del vector como eje integrador de las dos culturas, se describen las características sociales, ambientales y culturales que definen la enfermedad de Chagas en los wiwa y la posibilidad de su comprensión y manejo desde factores complementarios al modelo biomédico.

Conclusiones. Es necesario integrar de manera horizontal el conocimiento ancestral de las comunidades sobre los elementos ecológicos y culturales involucrados en la enfermedad de Chagas para el diseño de estrategias de manejo y control, más asertivas y respetuosas de las culturas.

$$
\bullet \bullet
$$

\section{Reporte de las barreras en la atención} de casos agudos de enfermedad de Chagas en el primer nivel de salud de Bucaramanga: conocimientos, actitudes y prácticas de los médicos de consulta externa del Instituto de Salud de Bucaramanga

Ligia Solano', William Aguilar', Gerardo Muñoz²

1 Instituto de Salud de Bucaramanga, Bucaramanga, Colombia

2 Universidad Industrial de Santander, Bucaramanga, Colombia

Introducción. La atención de casos agudos de la enfermedad de Chagas en Bucaramanga se ha visto confundida por afluencia masiva de fiebres a los consultorios del primer nivel. En el 2010 se reportaron 16.538 casos de fiebres no diferenciadas en el Instituto de Salud de Bucaramanga, que atiende el $30 \%$ de las consultas del primer nivel del régimen subsidiado. En lo corrido del 2011 se han registrado 7.000 consultas y un caso de Chagas agudo con diagnóstico errado durante cuatro semanas, como ha sucedido con el $100 \%$ de los casos anteriores. El objetivo del presente trabajo fue identificar las barreras en la atención en el primer nivel para el Chagas agudo.

Materiales y métodos. Se realizaron entrevistas personales con un cuestionario estructurado y a grupos focales de médicos del Instituto de Salud de Bucaramanga.

Resultados. Se entrevistaron cuarenta médicos en julio de 2011, la mayoría (90\%) con contratos permanentes, edad menor de 50 años (70\%), conformado por mujeres y hombres $(50 \%)$ y vinculación entre uno y 10 años (83 \%). Sus conocimientos sobre los casos agudos de enfermedad de Chagas han sido reforzados por los casos recientes, respondiendo como signos y 
síntomas "muy importantes", a la fiebre (70\%), la disnea de pequeños esfuerzos (78 \%) y el dolor torácico (70\%), aunque algunos signos, como el edema facial y el dolor de garganta, tuvieron bajos puntajes. Consideran herramientas diagnósticas relevantes la prueba ELISA (65\%), la radiografía (73 \%) y el electrocardiograma (75\%), pero desconocen la prueba de Strout (65\%), y $38 \%$ dan poca importancia a la gota gruesa. Consideran que los métodos diagnósticos no están a su alcance (85\%), les falta capacitación sobre el tema (85\%) y el diligenciamiento de las fichas epidemiológicas les quita tiempo (65\%).

Conclusiones. Se debe apoyar al primer nivel para evitar la falla en la diferenciación de las fiebres, permitir que los puestos de salud diagnostiquen enfermedad de Chagas y otras patologías asociadas a fiebres, reforzar el entrenamiento médico, actualizar y medir la observancia de las guías de atención, eliminar las barreras administrativas y sistematizar el diligenciamiento de las fichas epidemiológicas.

\section{Formación de promotores escolares para la creación de un programa de vigilancia de la enfermedad de Chagas con participación comunitaria en la zona rural del municipio de Mompós, departamento de Bolívar}

Omar Cantillo-Barraza1, Mariana Sanmartino², Jorge

Chica ${ }^{1}$, Carlos Ditta ${ }^{3}$, Julio Ramos ${ }^{4}$, Andrés Gómez-

Palacio $^{1}$, Omar Triana ${ }^{1}$

1 Grupo Biología y Control de Enfermedades Infecciosas, Universidad de Antioquia, Medellín, Colombia

2 Grupo de Didáctica de las Ciencias, Instituto de Física de Líquidos y Sistemas Biológicos, La Plata, Argentina

3 Institución Educativa Técnica Agropecuaria Ambiental, Tierrafirme, Colombia,

4 Institución Educativa Técnica Agropecuaria, La Rinconada, Colombia

Introducción. Los programas de control de la enfermedad de Chagas en casos mediados por vectores secundarios, se deben centrar en las especies nativas e incluir a la comunidad en riesgo para garantizar su contextualización y sostenibilidad. Sin embargo, la pobre participación social que caracteriza a la enfermedad de Chagas y la ausencia del componente educativo posterior a la intervención, se han convertido en la principal razón para la ausencia de programas de vigilancia con participación comunitaria.

El objetivo fue crear un programa de participación comunitaria mediante la formación de promotores escolares para la vigilancia de la enfermedad de Chagas, en la zona rural de Mompós.

Materiales y métodos. Las fases desarroladas fueron: I: evaluación del nivel óptimo de conocimiento sobre la enfermedad de Chagas en un grupo de estudiantes a formarse como promotores; II: capacitación teorico-práctica en parasitología, taxonomía y búsqueda de triatominos; III: uso de tecnología de la información y comunicación por los promotores para desarrollo, socialización y divulgación de las características locales de la enfermedad de Chagas, y IV: seguimiento y evaluación mediante encuestas

Resultados. La evaluación del nivel óptimo de conocimiento en la población mostró un nivel de conocimiento inferior al que debe tener la población de zonas endémicas. El material digital y didáctico fue construido a partir de la concepción de los estudiantes, como de conceptos adquiridos en la capacitación, fueron difundidos por los promotores por medio de tecnología de la información y comunicación y conversatorios locales. Esto permitió la multiplicación de la información en la comunidad y facilitó la participación en el programa de vigilancia vectorial. El seguimiento mostró que $86,3 \%$ de los estudiantes después de la intervención conocen sobre la enfermedad de Chagas y 93,4 \% reconoce los vectores. La gran mayoría de los escolares ha participado en el programa de vigilancia buscando insectos en sus casas y llevándolos al colegio.

Conclusión. La multiplicación de información por escolares mediante las tecnología de la información y comunicación promueven la participación en programas de vigilancia vectorial. 


\section{Epidemiología, vigilancia y control de enfermedades tropicales}

\section{Helmintiasis}

\section{Oncocercosis en Ecuador: reacción en cadena de la polimerasa para control de la infestación por Onchocerca volvulus en simúlidos}

Ángel Guevara, Yosselin Vicuña, Raquel Lovato, Eduardo Gómez, Mauricio Sauerbrey, Charles Katholi, Thomas Unnasch

Laboratorio de Parasitología Molecular, Centro de Biomedicina, Universidad Central del Ecuador, Quito, Ecuador

Programa Nacional de Oncocercosis, Ministerio de Salud Pública del Ecuador, Guayaquil, Ecuador Onchocerciasis Erradication Program for the Americas, Department of Global Health, Infectious Disease Research, College of Public Health, University of South Florida, Tampa, FLA, USA

Introducción. La oncocercosis es una de las mayores causas de ceguera en el mundo, originada por infección con el nematodo Onchocerca volvulus, y transmitida por insectos simúlidos. La enfermedad existe en áreas tropicales de África y América Latina; en Ecuador, el foco de Oncocercosis está en la provincia de Esmeraldas. Dentro del Programa para la Eliminación de la Oncocercosis en las Américas (OEPA), el Ministerio de Salud del Ecuador, a través del Programa para la Eliminación de la Oncocercosis, distribuye Ivermectina a las poblaciones afectadas; una de las maneras de evaluar la eliminación de la transmisión de $O$. volvulus es el análisis molecular del nematodo en los vectores de oncocercosis.

Materiales y métodos. Se recolectaron simúlidos en El Tigre y San Miguel, río Cayapas-Esmeraldas, en diferentes años; los simúlidos se identificaron y se procesaron para PCR-Ov150 y, se determinó el porcentaje de infestación.

Resultados. En 1995, se analizaron 2.250 simúlidos en El Tigre y 3.550 simúlidos en San Miguel con infestación de $72,7 \%$ y $62,0 \%$, en cada caso. En el 2000, en El Tigre se estudiaron 17.000 simúlidos y en San Miguel, 9.600 con 9 $\%$ y $1,1 \%$ de infestación, respectivamente. En el 2004, en El Tigre se estudiaron 10.100 simúlidos y 12.500 simúlidos en San Miguel con 6,0 \% y 0,8 $\%$ de infestación, en cada caso. En el 2008, en El Tigre se estudiaron 10.800 y en San Miguel, 11,863 simúlidos y no se detectó infestación en ninguno de ellos.

Conclusiones. Una de las metas del Programa de Eliminación de la Oncocercosis es obtener parámetros medibles para evidenciar la eliminación; uno de ellos, la supresión de la transmisión de $O$. volvulus se define como la ausencia de larvas de $O$. volvulus en la población de vectores. El método utilizado pare determinar este parámetro, PCR-ELISA Ov150, demuestra que en Ecuador la supresión de la transmisión es evidente.

Trabajo financiado por OEPA y el Ministerio de Salud Pública del Ecuador

\section{Lymphatic filariasis in Brazil: epidemiological situation and outlook for elimination}

Gilberto Fontes ${ }^{1,2}$, Anderson Leite ${ }^{1}$, Ana Rachel

Lima $^{1}$, Herbert Charles Barros ${ }^{3}$, Helen Freitas ${ }^{4}$, John

Ehrenberg $^{5}$, Eliana Rocha ${ }^{1,2}$

1 Universidade Federal de Alagoas, Maceio, Brasil

2 Universidade Federal de São João del Rei, Minas Gerais, Brasil

${ }^{3}$ Secretaria Municipal de Saúd, Maceió, Brasil

${ }^{4}$ Secretaria de Vigilância em Saúde, Ministério da Saúde, Brasilia, Brasil

${ }^{5}$ World Health Organization, Western Pacific Region

Introduction. To assess the current status of lymphatic filariasis in Brazil and the outlook for its elimination in the country before the year 2020, the target date established by WHO for global elimination.

Methods. A comprehensive bibliographic search for available studies and reports of filariasis epidemiology in Brazil was carried out and recovery publications analysed.

Results. From 1951 to 1958 a nationwide epidemiological study conducted in Brazil confirmed authochtonous transmission of Bancroftian filariasis in 11 cities of the country. Control measures led to a decline in parasite rates, and in 1980s only the cities of Belém in the Amazonian region (northern region) and Recife (northeastern region) were considered to be endemic. In the 1990s, foci of active transmission of lymphatic filariasis were described also in the cities of Maceió, Olinda, Jaboatão dos 
Guararapes, and Paulista, all in the northeastern coast of Brazil. Data provide evidence for the absence of microfilaremic subjects and infected mosquitoes in Belém, Salvador and Maceió in the past few years, attesting to the effectiveness of the measures adopted in these cities.

Conclusions. Currently, lymphatic filariasis is a public health problem in Brazil only in four cities of the metropolitan Recife region (northeastern coast). Efforts should be concentrated in these areas, with a view to eliminating the disease in the country.

\section{Avaliação da interrupção da transmissão da filariose linfática na cidade de Maceió, Estado de Alagoas, nordeste do Brasil através da determinação da prevalência de antigenemia por Wuchereria bancrofti (Cobbold, 1877).}

Gilberto Fontes ${ }^{1,2}$, Gabriela Vieira², Anderson Leite ${ }^{1}$, Ana Rachel Lima ${ }^{1}$, Ana Dalva Lima ${ }^{1}$, Lívia Texeira ${ }^{1}$, Rafael Santos ${ }^{1}$, Thiago Prado², Eliana Rocha ${ }^{1,2}$

1 Universidade Federal de Alagoas, Maceio, Brasil

2 Universidade Federal de São João Del Rei, Minas Gerais, Brasil

Introdução. Filariose linfática é problema de saúde pública mundial, e em 1997 a OMS considerou possível sua eliminação global até o ano 2020. Um dos critérios da OMS para determinar interrupção da transmissão da filariose linfática em uma área, é a pesquisa de antígenos sanguíneos de Wuchereria bancrofti em amostra de 3.000 crianças (6 a 10 anos) através do teste imunocromatografia rápida. A OMS considera que $<0,1 \%$ de antigenemia, a área analisada está livre de transmissão de filariose linfática.

Objetivo. Efetuar inquérito epidemiológico em Maceió, determinando a prevalência de portadores de antígenos de $W$. bancrofti, para verificar interrupção da transmissão da filariose linfática.

Materiais e métodos. Foi obtida amostra aleatória (a partir de quarteirões sorteados), de 3.000 crianças (6 a 10 anos), residentes nos 50 bairros da cidade. Para verificação da antigenemia foi utilizado o teste de imunocromatografia rápida. (Binax Now ${ }^{\circledR}$ Filariasis Immunochromatographic Test), técnica qualitativa baseada na busca de antígenos sanguíneos do parasito. A coleta sanguínea por punção digital foi obtida no período diurno, mas poderia também ser realizada no período noturno, pois não ocorre oscilação dos antígenos no sangue.
Resultados e discussão. Não foi detectada nenhuma amostra positiva entre as crianças examinadas, sendo $0,0 \%$ a prevalência de antígenos. Em Maceió, a filariose linfática apresentava recentemente uma distribuição focal em apenas três bairros centrais e contíguos (Feitosa, Jacintinho e Pitanguinha) e estudo semelhante de antigenemia realizado em 2003, detectou $10(0,33 \%)$ parasitados entre 3.000 crianças, mostrando, naquela época, haver transmissão da filariose linfáticana área.

O "Programa de eliminação da filariose linfática em Maceió", tendo como objetivos a detecção, tratamento e acompanhamento dos parasitados por período mínimo de dois anos, foi muito importante para a cidade apresentar, na atualidade, índice de prevalência de $0,0 \%$ de antigenemia.

Conclusão. Maceió pode ser considerada, de acordo com critérios da OMS, área livre de transmissão de $W$. bancrofti na atualidade.

Apoio financeiro: CNPq, Ministério da Saúde, FAPEAL, OPAS/OMS

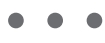

\section{Epidemiología de Trichinella spiralis} en Zacatecas, México, 2000-2010

María Alejandra Moreno-García1', Claudia MaldonadoTapia ${ }^{1}$, Ariadna Nayeli Ortiz-Reyna ${ }^{1}$, María del Refugio Vacío-De la Torre ${ }^{1}$, Vicente Berumen-De la Torre ${ }^{1}$, José Jesús Muñoz-Escobedo²

1 Unidad Académica de Ciencias Biológicas, Universidad Autónoma de Zacatecas, Zacatecas, México

2 Unidad Académica de Odontología, Cuerpo Académico de Biología Celular y Microbiología. Universidad Autónoma de Zacatecas, Zacatecas, México

Introducción. La triquinelosis es una zoonosis endémica en el estado de Zacatecas desde 1976, que afecta a mamíferos incluyendo al hombre.

El objetivo fue la detección de Trichinella spiralis en diferentes huéspedes en Zacatecas, México, de 2000 a 2010.

Materiales y métodos. Se logró el establecimiento del modelo experimental en ratón, cerdo y perro como control del ciclo vital del parásito y caracterización por las técnicas directas de compresión de tejido, y digestión artificial e indirectas de microinmunodifusión, Dot-ELISA e inmunoelectrotransferencia.

Se hizo el análisis de 100 diafragmas de cerdo del rastro municipal de Zacatecas y Jerez, 100 sueros de cerdos vivos de granja y 100 sueros de cerdos de traspatio; además, se hizo el análisis de 100 diafragmas y sueros de ratas domésticas 
del basurero municipal de Zacatecas, el análisis de 100 lenguas de perros domésticos y de 1.250 sueros de humano del laboratorio del Sector Salud de Zacatecas. Se hizo el diagnóstico por técnicas directas e indirectas.

Resultados. En los modelos experimentales se estableció el ciclo vital del parásito y por técnicas directa e indirectas se estableció el diagnóstico.

En tejido de cerdo, por técnica directa, se obtuvo un resultado positivo del rastro municipal de Jerez y, por técnica indirecta, 10 positivos; de los sueros de traspatio, se obtuvieron 5 resultados positivos y 5 positivos de granja; en rata doméstica del basurero municipal de Zacatecas, se detectaron 3 positivas por técnicas directas y 3 perros positivos por la mismas técnicas; en sueros de humanos se detectaron 12 resultados positivos por técnicas indirectas con predominio del triplete de 42,45 y 48 kDa por inmunoelectrotrasferencia.

Conclusiones. Se detectó $T$. spiralis, en los huéspedes que permiten su permanencia (rata, cerdo) y en el hombre y su mascota de excelencia, el perro, siendo más efectivas las técnicas indirectas; el diagnóstico se puede realizar desde la segunda semana de infección por técnica indirecta de inmunoelectrotransferencia en el modelo experimental.

\section{Estudio epidemiológico de la infección por Strongyloides stercolaris y Trypanosoma cruzi en una localidad del norte de la provincia de Salta, departamento de Orán, Argentina}

Rubén Cimino ${ }^{1,2}$, Noelia Floridia ${ }^{1}$, Paola Vargas ${ }^{1}$, Silvana Cajal'², Marisa Juarez², Adriana Di Paolo², Norma Acosta², Carlos Villalpando², Eugenia Socias ${ }^{3}$, Florencia Veja Benedetti ${ }^{1}$, Thomas Nutman ${ }^{4}$, Julio Nasser $^{1,2}$, Alejandro Krolewiecki ${ }^{2,5}$

1 Cátedra de Química Biológica, Universidad Nacional de Salta, Argentina

2 Instituto de Investigaciones de Enfermedades Tropicales, Sede Orán, Universidad Nacional de Salta, Argentina

3 Fundación Mundo Sano

${ }^{4}$ Laboratory of Parasitic Disease, National Institutes of Allergy and infectious diseases, Bethesda, MD, USA

5 CONICET

Introducción. El norte de Argentina, región subtropical, es zona endémica para enfermedades parasitarias como las geohelmintiasis, la enfermedad de Chagas y la leishmaniasis. Con el objetivo de desarrollar estrategias de intervención sanitaria se estudio la situación epidemiológica de la infección por Strongyloides stercolaris y Trypanosoma cruzi, en una localidad en el norte de la provincia de Salta.

Materiales y métodos. Se realizó un estudio transversal en un asentamiento (22 $53^{\prime} 60^{\prime \prime} \mathrm{S}$, $\left.64^{\circ} 20^{\prime} 06^{\prime \prime} \mathrm{W}\right)$ que cuenta con una población de 618 habitantes. Se tomaron al azar muestras de sangre a 80 personas. Se realizaron estudios serológicos para el diagnóstico de la infección de $S$. stercolaris (ELISA-NIE) y T. cruzi (ELISA Chagastest Wiener Lab recombinante y ELISA-Ag1).

Resultados. La seroprevalencia de S. stercolaris fue de 31,25 \% (25/80) con $\mathrm{IC}_{95 \%}$ : 20,47 - 42,03. La edad media más la desviación estándar de los seropositivos fue de $32,85 \pm 20,8$. La seroprevalencia de la enfermedad de Chagas fue de 22,5\% $(18 / 80)\left(\mathrm{IC}_{95 \%}\right.$ : 12,72-32,27). La edad media más la desviación estándar de los seropositivos fue de $38,6 \pm 18,21$. El 22,22 \% de los infectados por $T$. cruzi eran menores de 20 años. La coinfección $S$. stercolaris- $T$. cruzi fue de 7,5 \% (6/80).

Conclusión. Según esta situación, la intervención sanitaria implicaría el tratamiento puntual para la enfermedad de Chagas en aquellos posible y tratamiento masivo en la comunidad para la estrongiloidiasis, además de la interrupción y control en la transmisión de la geohelmintiasis.

\section{Presunto brote humano causado por Trichinella T12 en la región de la Patagonia}

Silvio Jesús Krivokapich ${ }^{1}$, Patricia Arbusti ${ }^{1}$, Cinthia

Lorena Gonzalez-Prous ${ }^{1}$, Graciana Mabel Gatti ${ }^{1}$, Luisa

Saldia ${ }^{2}$

1 Departamento de Parasitología, Instituto Nacional de Enfermedades Infecciosas, Administración Nacional de Laboratorios e Institutos de Salud, Dr. Carlos G. Malbrán, Buenos Aires, Argentina

2 Hospital Dr. José Formenti, Ministerio de Salud Pública, El Calafate, Santa Cruz, Argentina

Introducción. La triquinelosis es una zoonosis parasitaria causada por los miembros del género Trichinella, donde Trichinella T12 es el único genotipo silvestre de Suramérica que fue descrito recientemente en un Puma (Puma concolor) en Argentina.

El objetivo del presente estudio fue la descripción de un presunto brote por este parásito autóctono en la región de la Patagonia.

Materiales y métodos. Ocho adultos de sexo masculino que consumieron carne de puma infectada con Trichinella T12 el 11 de junio de 2008 en El Calafate, fueron examinados por personal 
médico e inmediatamente tratados con albendazol (10 mg/kg aldía) durante una semana. El alimento se analizó por digestión artificial estándar y, posteriormente, seis ratones CF-1 se inocularon con 300 larvas musculares viables. Se obtuvieron muestras de suero de todos las personas implicadas 14 días después del consumo, para la detección de anticuerpos mediante la técnica ELISA y confirmación por Western blot. Cuatro pacientes retornaron a la segunda muestra 49 días más tarde y únicamente un paciente completó las tres muestras a los 126 días.

Resultados. La intensidad de la infección en el alimento examinado fue de 5 larvas por gramo y todos los ratones inoculados resultaron infectados. Ninguno de los pacientes evidenció signos ni síntomas clínicos relacionados con la triquinelosis. De los ocho analizados, tres fueron positivos en las pruebas serológicas de la primera y segunda muestra de suero. El único paciente que completó las tres tomas de muestras de sueros siempre se mostró reactivo.

Conclusión. Los resultados obtenidos sugieren la infección por ingestión de carne parasitada de puma, con larvas viables e infectivas de Trichinella T12, de tres de los ocho pacientes implicados, en quines la ausencia de síntomas clínicos estaría dada por el tratamiento antihelmíntico temprano. Alternativamente, los anticuerpos detectados corresponderían a una infección previa por Trichinella sp. 


\section{Epidemiología, vigilancia y control de enfermedades tropicales}

LEISHMANIASIS

\section{Factores ambientales de riesgo en la transmisión de leishmaniasis cutánea americana en el municipio de Chaparral, Tolima}

Carlos Valderrama ${ }^{1}$, Neal Alexander ${ }^{2}$, Cristina Ferro ${ }^{3}$,

Clara B. Ocampo 2

1 Universidad Icesi, Cali, Colombia

2 Centro Internacional de Entrenamiento e Investigaciones Médica, Cali, Colombia

3 Instituto Nacional de Salud, Bogotá, D.C., Colombia

Introducción. El análisis espacial permite evaluar el papel de las variables ambientales para explicar la distribución de los vectores y los cambios ambientales que pueden observarse en las enfermedades transmitidas por vectores. El reciente brote de leishmaniasis cutánea americana en el municipio de Chaparral (Tolima) (2004-2006) ha sido evaluado desde esta perspectiva para poder identificar las variables que mejor explican el riesgo de transmisión de la enfermedad.

Metodología. Mediante el análisis multitemporal de coberturas vegetales en imágenes LandSat, variables climáticas (BioClim) y topográficas (SRTM-DEM $90 \mathrm{~m}$ ) en un sistema de información geográfica, se evaluó el brote a escala del paisaje (1:100.000) para todo el municipio. A una escala más fina, de peridomicilio, se evaluaron las coberturas en 8 "transectos" con estaciones cada $10 \mathrm{~m}$, radiales en un círculo de $100 \mathrm{~m}$ de radio alrededor de las viviendas evaluadas $(n=43)$. Esta última fue evaluada en dos veredas con incidencias contrastantes de leishmaniasis pero con rango de elevación similares.

Resultados. Se encontró que las variables que más afectan la incidencia de la transmisión de leishmaniasis cutánea americana en Chaparral son la presencia de una alta proporción de bosque y rastrojo (un incremento de 2,6 \% por cada porcentaje de aumento de bosque) y una temperatura promedio $20,6{ }^{\circ} \mathrm{C}$ que se asocia con elevaciones entre los 1.400 y los $1.600 \mathrm{~m}$. No se observaron cambios significativos en la cobertura vegetal en los últimos 20 años. La vereda con alta incidencia presentó una cobertura de bosque mayor (39,3\%) que la vereda con baja incidencia $(8,7 \%)$. La presencia de bosque a menos de $30 \mathrm{~m}$ de la vivienda presentó mayor riesgo de presencia del vector.

Conclusiones. Las variables ambientales afectan el riesgo de transmisión de la leishmaniasis cutánea americana. El entendimiento del efecto de estos factores sobre la transmisión de la leishmaniasis cutánea americana permitirá la elaboración de mapas de riesgo para esta enfermedad.

\section{Caracterización ecoepidemiológica de la leishmaniasis lutánea en cuatro municipios del oriente del departamento de Caldas}

Horacio Cadena, Daniel Agudelo, Luz A. Acosta, Iván D. Vélez, Andrés F. Vélez

Programa de Estudio y Control de Enfermedades Tropicales, Universidad de Antioquia, Medellín, Colombia

Introducción. La leishmaniasis es un problema creciente de salud pública en Colombia, con un aumento inusitado de casos desde el 2003, debido principalmente a la adaptación del vector al entorno humano, a la aparición de nuevos focos y a la urbanización del ciclo de transmisión.

El departamento de Caldas registró 107 casos en el 2009 en los municipios de Marquetalia, Norcasia, Samaná y Victoria, y pasó a 294 en el 2010. Este brote epidémico fue estudiado mediante la aplicación del método ecoepidemiológico que permitió realizar evaluación entomológica, tipificación de las cepas de Leishmania circulantes, educación a la comunidad y capacitación al personal de salud de cada municipio.

Metodología. La captura de flebotominos se realizó con trampas CDC en el intradomicilio y peridomicilio. La exposición de la población al parásito se determinó con la prueba de Montenegro. El aislamiento de las cepas de Leishmania se realizó en medio NNN y se identificó por PCRRFLP.

Resultados. Se capturaron 389 flebotominos, correspondientes a 14 especies, de los cuales, $37 \%(n=145)$ y $31 \%(n=122)$ fueron Lutzomyia trapidoi y Lu. yuilli, respectivamente. Por búsqueda activa se atendieron 57 pacientes, con 35 positivos $(61 \%)$ para leishmaniasis. La prueba 
de Montenegro se aplicó a 171 personas y en 54 (32\%) resultó con induración igual a 5 mm o mayor. Las cepas circulantes corresponden a Leishmania panamensis y $L$. braziliensis. Se dictaron charlas educativas a 496 integrantes del personal médico y comunidades de los cuatro municipios.

Discusión. La captura de vectores comprobados de leishmaniasis en el intradomicilio y peridomicilio, y el registro de niños con una prueba Montenegro positiva, demuestran la domiciliación del vector en la región, lo que implica un alto riesgo de infección para la población en estas localidades. Se recomienda la educación continua al personal médico y a la comunidad y el uso de toldillos.

\section{Transferencia tecnológica para el mejoramiento de la vigilancia y control de la leishmaniasis en Centroamérica}

Karina Mondragón-Shem, Horacio Cadena, Andrés F.

Vélez, Liliana López, Luz A. Acosta, Daniel Agudelo,

Carlos Muskus, Rafael Villareal, Ronald Peláez, Mónica

Zuleta, Karina Salgado, Iván D. Vélez

Programa de Estudio y Control de Enfermedades

Tropicales, Universidad de Antioquia, Medellín,

Colombia

Introducción. La leishmaniasis es una enfermedad desatendida que afecta a millones de personas en las regiones tropicales y subtropicales del mundo. Uno de los mayores problemas que enfrentan los países endémicos, es la falta de conocimientos y entrenamiento requeridos para el estudio adecuado de los focos de transmisión.

El objetivo fue llevar a cabo la transferencia de tecnología para la capacitación multidisciplinaria al personal de salud en la metodología de la evaluación ecoepidemiológica de los focos de leishmaniasis en Guatemala, El Salvador, Panamá, Nicaragua y Costa Rica durante el segundo semestre del 2010.

Metodología. Se visitó cada país, llevando a cabo capacitaciones y el apoyo al estudio de un foco importante de leishmaniasis, con atención de pacientes, charlas a la comunidad, estudios entomológicos, búsqueda de reservorios domésticos y georreferenciación. Además, se elaboró el "Manual de diagnóstico y control de la leishmaniasis en Centroamérica" para ser entregado al personal de salud. Posteriormente, se realizaron dos cursos: el primero sobre identificación molecular de Leishmania spp. en el Programa de Estudio y Control de Enfermedades Tropicales, y el segundo sobre sistemas de información geográfica por medio de una plataforma de Moodle de la Universidad de Antioquia, en el que aprendieron a utilizar el SIGEpi, software de análisis epidemiológico desarrollado por la OPS.

Resultados y conclusiones. Se capacitaron más de 200 personas entre funcionarios y personal de salud de cada país, lográndose el aislamiento e identificación de especies de Leishmania a partir de las muestras de pacientes, y se identificaron los flebotomíneos recolectados para cada país encontrándose nuevos reportes entre los 2.910 individuos colectados.

La falta de un programa nacional para la leishmaniasis se traduce en un alto subregistro, y la enfermedad continúa siendo desatendida en estos países. Es necesario un mayor entrenamiento en los países endémicos, y mantener o actualizar las redes de colaboración de leishmaniasis que fueron iniciadas durante este programa.

\section{Estudio ecoepidemiológico de la leishmaniosis en el Darién colombiano}

Lina María Carrillo, ${ }^{1,2}$, Horacio Cadena', Luz Adriana Acosta ${ }^{1}$, Karina Mondragón-Shem ${ }^{1}$, Andrés Vélez ${ }^{1}, \mathrm{D}$. Agudelo $^{1}$, J. E. Pérez ${ }^{1}$, J. Trujilloº , Iván Darío Vélez ${ }^{1}$

1 Programa de Estudio y Control de Enfermedades Tropicales, Universidad de Antioquia, Medellín, Colombia

2 Facultad de Ciencias Agrarias, Universidad de Antioquia, Medellín, Colombia

Introducción. Los focos naturales de infección de leishmaniasis han sido estudiados abordando diferentes metodologías. Este estudio propuso utilizar un método holístico que permitiera una visión general de la enfermedad para tener más y mejores opciones de prevención y control, en una zona de alto impacto turístico.

Metodología. El estudio se llevó a cabo en la región del Darién chocoano, en la zona rural del municipio de Acandí, con 5.190 habitantes.

Se desarrollaron las siguientes actividades: 1) captura de fauna de flebotominos en reposo y con trampas CDC, Shannon y adhesivas; 2) caracterización clínica de la enfermedad realizando diagnóstico e identificación de cepas por métodos directos, IFI, PCR e isoenzimas; 3) aplicación de la prueba de Montenegro; 4) estudio de reservorios domésticos y silvestres; 5) estudio de concepciones, actitudes y prácticas de la enfermedad combinando métodos cuantitativos y cualitativos; 6) transferencia de tecnología capacitando al personal de salud y realizando educación primaria en salud a la comunidad.

Resultados y discusión. Se capturaron 4.804 
flebotominos pertenecientes a 25 especies, y se identificó como vector principal a Lutzomyia panamensis, con una alta domiciliación, especialmente en las zonas con mayor grado de urbanización. Otras especies en orden de abundancia fueron: Lu. carpenteri, Lu. trinidadensis, Lu. triramula y Lu. trapidoi. Se registró $L u$. cayennensis cayennensis como nueva especie para el departamento del Chocó.

Se identificó a Leishmania panamensis como agente causal de las lesiones cutáneas y mucosa, y se tipificaron dos clones diferentes por medio de isoenzimas Todos los casos se trataron exitosamente con miltefosina. Se encontró que las concepciones y las costumbres de la población favorecen la presentación de la enfermedad en la zona. Se propone como posible reservorio de enlace a Didelphis marsupialis y a Canis familiaris como huésped accidental.

Conclusión. El abordaje metodológico de este estudio permitió caracterizar los determinantes de la dinámica del ciclo de transmisión de la leishmaniasis cutánea para el diseño de un programa de prevención y control en esta zona del país.

\section{Evaluación ecoepidemiológica de la leishmaniasis en El Carmen de Bolívar}

Luis Cortes, Ramiro Pereira

Unidad de Entomologia de Bolívar, Laboratorio de Salud Pública, Cartagena, Colombia

Introducción. El Carmen de Bolívar presenta la mayorincidenciadeleishmaniasisvisceralycutánea, aportando el $53 \%$ de los casos de leishmaniasis cutánea y el $100 \%$ de los casos de leishmaniasis visceral registrada en el departamento de Bolívar. Durante el período estudiado se presentó una mortalidad del $16 \%$ de niños menores de cinco años por leishmaniasis visceral.

El objetivo principal fue realizar una evaluación ecoepidemiológica para ampliar los conocimientos epidemiológicos, ecológicos, sociales y culturales de la leishmaniasis en el municipio que permitieran implementar una estrategia integrada de prevención y control de la enfermedad.

Materiales y métodos. Se analizaron todos los datos epidemiológicos, serológicos y entomológicos obtenidos en los diferentes estudios de brotes y en las investigaciones realizadas durante los años 2002 a 2008 en el municipio de El Carmen de Bolívar; estos datos se tabularon y analizaron contra variables independientes con Excelß y Statistic®, versión 6.0 .

Resultados. Se establecieron los patrones de comportamiento de las especies de Lutzomyia de importancia médica, se determinó la prevalencia de infección de los reservorios, se caracterizaron epidemiológicamente las zonas por intervenir y se desarrolló un programa de educación comunitaria, un plan de tratamiento y de nutrición a niños menores de cinco años en las veredas prioritarias, además de capacitaciones en el manejo de esta enfermedad a profesionales de la salud. Durante la aplicación de la estrategia se ha obtenido una reducción de $76 \%$ de los casos de leishmaniosis visceral y una disminución de la tasa de mortalidad del $100 \%$.

Conclusiones. La estrategia integral ha dado resultados promisorios. Se comprueba que los toldillos y las cortinas impregnadas brindan unas herramientas de protección a las picaduras de flebótomos dentro de las viviendas, actuando en forma de barrera e insecticida contra Lutzomyia spp. con comportamientos exofílicos y endofágicos; sin embargo, no están dentro de los saberes y costumbres de los pobladores de estas zonas intervenidas.

\section{Nuevos aportes de la epidemiología molecular en Venezuela: Lutzomyia migonei y el perro (Canis familiaris) mantienen dos ciclos epidemiológicos de leishmaniasis cutánea en la región andina*}

M. Dora Feliciangeli ${ }^{1}$, Patricia Villegas ${ }^{1}$, Annhy

Torrellas ${ }^{1}$, Arturo Bravo ${ }^{1}$, Héctor de Lima ${ }^{1}$, José

Carrero², Olinda Delgado ${ }^{3}$, Israel $\mathrm{Cruz}^{4}$, Carmen

Cañavate $^{4}$, Elisabeth Ferrer ${ }^{1}$, Michael Miles ${ }^{5}$

1 Universidad de Carabobo, BIOMED, Facultad de Ciencias de la Salud, sede Aragua, Maracay, Venezuela

2 Instituto de Biomedicina, Ministerio del Poder Popular para la Salud, Caracas, Venezuela

3 Servicio de Dermatología Sanitaria, Tovar, Venezuela

4 Instituto de Medicina Tropical, Universidad Central de Venezuela, Caracas, Venezuela

5 Instituto de Salud Carlos III, Madrid, España

6 London School of Hygiene and Tropical Medicine, London, UK

Introducción. La epidemiología molecular brinda herramientas valiosas para el entendimiento de la dinámica de la trasmisión de las parasitosis, la cual es clave para la aplicación de medidas oportunas de prevención y control.

Objetivo. Investigar los ciclos epidemiológicos de la leishmaniasis cutánea en un foco de alta endemia en Venezuela. 
Materiales y métodos. De noviembre de 2007 a marzo de2009 se realizó un estudio longitudinal de la fauna de flebótomos usando trampas CDC y de Shannon en El Carrizal, municipio Tovar

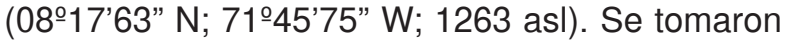
biopsias cutáneas de los pacientes que acudieron al Servicio de Dermatología para atención médica. Igualmente, se obtuvieron muestras de sangre de perros de El Carrizal. Para la detección de la infección de los parásitos se utilizó la técnica PCRITS y PCR-anidada y por medio de la reacción RFLP, utilizando Haelll, se realizó su identificación.

Resultados. Se capturaron 10 especies de flebótomos. Lutzomyia youngi y Lu. spinicrassa (grupo Verrucarum), constituyeron el $90 \%$ de la población total; Lu. migonei se recolectó en menor cantidad. Se detectó la presencia de Leishmania mexicana y $L$. guyanensis en grupos (pools) de Lu. Migonei. En los pacientes se detectó la presencia de ADN de L. braziliensis, L. guyanensis y $L$. Mexicana, y en perros de $L$. guyanensis y $L$. mexicana.

Conclusiones. Se describieron por primera vez en Venezuela dos ciclos epidemiológicos de transmisión de Leishmania spp. en los cuales Lu. migonei esel vector y el perro juega un papel importante como reservorio. Todavía se desconoce en esta área de estudio, el ciclo de L. braziliensis. Proyectos STREP-FP6-INCO-CT2005-015407 y FONACIT-MC 2008000911-2.

* Presentado en ISOPS 7, Kusadasi, Turquía, abril de 2011

$$
\text { - }
$$

\section{Estudio epidemiológico de dos focos de leishmaniasis cutánea en las veredas Guineo Bajo, Apartadó, y Aguas Claras, Turbo, Antioquia}

Margarita Arboleda, Gabriel Jaime Parra, Natalia Molina, Luz Yaned Usuga

Instituto Colombiano de Medicina Tropical, Universidad CES, Apartadó y Medellín, Colombia, y Facultad de Medicina, Universidad CES, Medellín, Colombia

Introducción. Urabá es una región endémica para leishmaniasis cutánea y, últimamente, se insinúa un aumento en la incidencia de la enfermedad.

El presente estudio pretendió caracterizar clínica, epidemiológica y entomológicamente dos focos de leishmaniasis cutánea en las veredas El Guineo de Apartadó y Aguas Claras del municipio de Turbo.

Materiales y métodos. Se llevó a cabo un estudio descriptivo de foco eco-epidemiológico con determinación de anticuerpos para leishmania, prueba de Montenegro y estudio de las lesiones sugestivas para la población y muestreo entomológico de las viviendas con casos positivos en los dos últimos años, utilizando trampas tipo CDC y Shannon en el intradomicilio, peridomicilio y extradomicilio.

Resultados. En la vereda El Guineo se encontró una prevalencia de lesiones activas por Leishmania panamensis de 2,77 \% (2/72), anticuerpos positivos en $77,2 \%(51 / 66)$, prueba de Montenegro mayor de $5 \mathrm{~mm}$ en 91,6 \% (44/48) y $30 \%(21 / 70)$ de cicatrices antiguas compatibles con Leishmaniasis. En la vereda Aguas Claras se encontró una prevalencia de lesiones activas por $L$. panamensis de $4,0 \%$ (2/50), anticuerpos positivos en $77,2 \%$ (51/66), prueba de Montenegro mayor de $5 \mathrm{~mm}$ en $94,7 \%(36 / 38)$, y $16,3 \%(8 / 49)$ de cicatrices antiguas compatibles con leishmaniasis. En la vereda El Guineo se encontraron las especies Lutzomya panamensis, Lu. trapidoi y Lu. dysponeta. Lutzomyia panamensis representó el $72,7 \%$ de los vectores recolectados en el intradomicilio y peridomicilio. En la vereda Aguas Claras se encontraron las siguientes especies de vectores: Lu. trapidoi, Lu. panamensis, Lu. barretoi y Lu. tortura. Lu. panamensis fue la especie más frecuente $(50 \%)$ y se capturó en el peridomicilio.

Conclusiones. Se confirma la elevada endemia de la leishmaniasis cutánea en estas veredas. Lutzomyia barretoi se registra por primera vez para la región de Urabá; Lu. dysponeta y Lu. tortura se reportan por primera vez para el departamento de Antioquia. Lutzomyia panamensis puede ser la especie responsable de la transmisión de la leishmaniasis cutánea en la zona de estudio.

\section{Relationship between anaemia and clinical forms in canine leishmaniasis}

Raul Ribeiro1, Sydnei Silva², Marilene Michalick², Frédéric Frézard ${ }^{2}$

1 Universidade Federal do Recôncavo da Bahia, Bahia, Brasil

2 Universidade Federal de Minas Gerais, Minas Gerais, Brasil

Introduction. Leishmaniasis is a group of illnesses transmitted by sand flies that affects domestic and wild animals, as well as humans. Dogs are considered the main reservoir of the etiological agent of visceral leishmaniasis in the urban context and one of the targets in the control strategy. The clinical characteristics of canine leishmaniasis vary from animals that are apparently healthy to animals with severe disease, with involvement of multiple 
organs and a wide variety of laboratory findings. The aim of this study was to identify the main biochemical and hematological findings in dogs naturally infected with Leishmania (Leishmania) chagasi and associate the laboratory changes with clinical forms of canine leishmaniasis.

Materials and methods. Blood samples for biochemical and hematological evaluation were taken from the cephalic vein of fifty one mongrel dogs, fifteen uninfected (control group) and thirty six infected, which were classified clinically in three groups: asymptomatic $(n=12)$, oligosymptomatic $(n=12)$ and symptomatic $(n=12)$. Dogs were identified during an epidemiological survey of canine leishmaniasis. The infection was confirmed by serology (indirect immunofluorescence antibody test, IFAT; enzyme-linked immunosorbent assay, ELISA) and parasitological diagnosis.
Results. Total protein, urea, a-globulin 2, globulin and albumin/globulin ratio (A-G ratio) levels of leishmaniasis groups were out of reference interval and significantly different when compared to healthy dogs. Anaemia was registered in groups with clinical signs of canine leishmaniasis and statistical analyses indicated significantly higher frequency of erythrogram disorders in these groups than in asymptomatic group. A significant association was observed between the anaemia and the appearance of the symptoms, where dogs with highest erythrogram values showed the better clinical condition.

Conclusions. Our results provide additional evidence that the clinical form in canineleishmaniasis may reflect the erythrogram status of the animals and the A-G ratio is a reliable biochemical marker for diagnosis and therapeutic monitoring. 